\title{
Adjunctive tests cannot replace scalpel biopsy for oral cancer diagnosis
}

\author{
Abstracted from \\ Cochrane Database Syst Rev 2015; 5: CD010276. DOI:10.1002/14651858.CD010276.pub2. \\ Address for correspondence: Luisa Fernandez Mauleffinch, Managing Editor, \\ Cochrane Oral Health Group, School of Dentistry, The University of Manchester, \\ JR Moore Building, Oxford Road, Manchester, M13 9PL, UK. \\ E-mail: luisa.fernandez@manchester.ac.uk
}

Macey R, Walsh T, Brocklehurst P, Kerr AR, Liu JL, Lingen MW, Ogden GR, Warnakulasuriya S, Scully C.

Diagnostic tests for oral cancer and potentially malignant disorders in patients presenting with clinically evident lesions.

\section{Question: What is the diagnostic accuracy of tests for the detection of oral cancer and potentially malignant disorders (PMD) of the lip and oral cavity, in people presenting with clinically evident lesions?}

Data sources Medline, the Cochrane Diagnostic Test Accuracy Studies Register, the Cochrane Oral Health Group's Trials Register, Embase and MEDION.

Study selection Two of the reviewers independently assessed titles abstracts and extracted data. Cross-sectional diagnostic test accuracy studies (or consecutive series) and randomised studies of diagnostic test accuracy that reported the diagnostic test accuracy of vital staining, oral cytology, light-based detection and oral spectroscopy, blood or saliva analysis used as an adjunct to conventional oral examination in detecting PMD or oral squamous cell carcinoma of the lip or oral cavity were considered. Scalpel, punch or fine needle aspiration biopsy with histological diagnosis was the reference test. Data extraction and synthesis Study quality was assessed using a modified version of QUADAS-2. Meta-analysis was used to combine the results of studies for each index test using the bivariate approach to estimate the expected values of sensitivity and specificity. Metaregressionanalysis was undertaken to explore possible sources of heterogeneity.

Results Forty-one studies, recruiting 4002 patients were included. No single study could be classified as being at low risk of bias across

\begin{tabular}{|l|l|l|l|}
\hline & $\begin{array}{l}\text { No. of } \\
\text { studies in } \\
\text { meta-analysis }\end{array}$ & $\begin{array}{l}\text { Sensitivity } \\
(\mathbf{9 5 \%} \mathbf{C l})\end{array}$ & $\begin{array}{l}\text { Specificity } \\
(\mathbf{9 5} \% \mathbf{C l})\end{array}$ \\
\hline Vital staining & 14 & $\begin{array}{l}0.84(0.74 \text { to } \\
0.90)\end{array}$ & $\begin{array}{l}0.70 \\
(0.59 \text { to } 0.79)\end{array}$ \\
\hline Cytology & 12 & $\begin{array}{l}0.91(0.81 \text { to } \\
0.96)\end{array}$ & $\begin{array}{l}0.91 \\
(0.81 \text { to } 0.95)\end{array}$ \\
\hline $\begin{array}{l}\text { Light-based } \\
\text { detection }\end{array}$ & 11 & $\begin{array}{l}0.91(0.77 \text { to } \\
0.97)\end{array}$ & $\begin{array}{l}0.58 \\
(0.22 \text { to } 0.87)\end{array}$ \\
\hline
\end{tabular}

This paper is based on a Cochrane Review published in the Cochrane Library 2015, issue 5 (see www.thecochranelibrary.com for information). Cochrane Reviews are regularly updated as new evidence emerges and in response to feedback, and the Cochrane Library should be consulted for the most recent version of the review. all domains. Fourteen studies evaluated vital staining, 13 studies oral cytology and 13 studies light-based detection or oral spectroscopy, while six studies assessed two combined index tests. There were no eligible diagnostic accuracy studies evaluating blood or salivary sample analysis.

Conclusions The overall quality of the included studies was poor. None of the adjunctive tests can be recommended as a replacement for the currently used standard of a scalpel biopsy and histological assessment. Given the relatively high values of the summary estimates of sensitivity and specificity for cytology, this would appear to offer the most potential. Combined adjunctive tests involving cytology warrant further investigation.

\section{Commentary}

Globally oral cancer is the sixth most common cancer but unlike many cancers its incidence is increasing. ${ }^{1}$ Here in the UK the incidence of oral cancer has risen by a third in the last decade and in 2011 around 6800 people a day were diagnosed with oral cancer. ${ }^{2}$ The incidence is strongly associated with social inequality ${ }^{3}$ and diagnosis is often at a late stage when the prognosis is poor and the risks of significant morbidity and mortality are substantially higher. ${ }^{4}$ While treatment and management of oral cancer has improved in the recent decade ${ }^{5,6}$ five-year survival after diagnosis has remained relatively static over the past 30 years, although there is a site-to-site variation. ${ }^{2}$

The aim of this review was to estimate the diagnostic accuracy of a number of tests that can be used as adjuncts to oral examination to detect squamous cell carcinoma (OSCC) and potentially malignant disorders (PMD). These tests were; vital staining (toluidine blue), oral cytology, light-based detection and blood and saliva analysis.

A detailed database search was conducted and the authors included diagnostic test accuracy studies that used scalpel, punch or fine needle aspiration biopsy with histological diagnosis as a gold (reference) standard. Study quality was assessed using the QUADAS-2.7 Forty one studies were included with a majority (30) assessing just a single test on a single sample; the other eleven assessed multiple tests in the same sample. No studies were included of blood and saliva analysis. While all the included studies used an appropriate reference standard there was a lack of detail and in three studies the reference test was not independent of the index test. Overall none of the studies was considered to be at low risk of bias across all of the quality domains. 
The estimates of sensitivity and specificity for the three index tests included are shown in the table and of the three modalities the estimates for oral cytology were the highest. However, as the authors note in the discussion, these findings should be interpreted with caution. This is because the overall quality of the studies is poor and all the included studies were conducted in a secondary care environment and as such these findings are unlikely to have a direct read across to the primary care situation.

This review together with the related Cochrane reviews ${ }^{8,9}$ provide very helpful summaries of the evidence base in this area at a time when there is an increasing awareness of the problem of oral cancer and pressure for a screening programme in primary care. At the moment in the UK the National Screening Committee (NSC) is reviewing oral cancer screening in adults and it is currently in consultation until September. The review is taking place against the NSC criteria, and when oral cancer screening was last reviewed in 2010 it was not recommended. The latest expert review is available on the NSC website and again many of the NSC criteria are not met so it is unlikely to be recommended. ${ }^{10}$

As the NSC review and this current review notes, the natural history of oral cancer is not fully understood; not all PMDs undergo malignant transformation and oral cancer can develop from lesions in which epithelial dysplasia was not previously diagnosed. Also at this time neither conventional oral screening ${ }^{8}$ nor the adjunctive tests discussed in this current review are sufficiently accurate to be used as a screening test in a formal national screening programme.

However, important risk factors in the development of oral cancer; tobacco, betel quid, alcohol, age, gender and sunlight are known, ${ }^{9}$ and the dental team should regularly highlight these to their patients. They should also regularly examine the entire mouth and raise their index of suspicion regarding any lesion to prevent delays in onward referral.

\section{Derek Richards}

Centre for Evidence-based Dentistry, Dental Health Services Research Unit, University of Dundee, Dundee, Scotland.

1. Warnakulasuriya S. Global epidemiology of oral and oropharyngeal cancer. Oral Oncol. 2009; 45: 309-316.

2. Cancer Research UK, (http://www.cancerresearchuk.org/health-professional/cancerstatistics/statistics-by-cancer-type/oral-cancer), Accessed [June] [2015].

3. Conway DI, McMahon AD, Smith K, et al. Components of socioeconomic risk associated with head and neck cancer: a population-based case-control study in Scotland. Br J Oral Maxillofac Surg. 2010; 48: 11-17.

4. Rusthoven KE, Raben D, Song II, Kane M, Altoos TA, Chen C. Survival and patterns of relapse in patients with oral tongue cancer. J Oral Maxillofac Surg. 2010; 68: 584-589.

5. Glenny AM, Furness S, Worthington HV, et al. Interventions for the treatment of oral cavity and oropharyngeal cancer: radiotherapy. Cochrane Database Syst Rev. 2010; 8: [DOI: 10.1002/14651858.CD006387.pub2]

6. Furness S, Glenny AM, Worthington HV, et al. Interventions for the treatment of oral cavity and oropharyngeal cancer: chemotherapy. Cochrane Database Syst Rev. 2011; 13: [DOI: 10.1002/14651858.CD006386.pub3]

7. QUADAS-2 http://www.bris.ac.uk/quadas/quadas-2/ (accessed June 2015)

8. Walsh T, Liu JLY, Brocklehurst $P$, et al. Clinical assessment to screen for the detection of oral cavity cancer and potentially malignant disorders in apparently healthy adults. Cochrane Database Syst Rev. 2013; 11: Art. No. CD010173. DOI: 10.1002/14651858.CD010173.pub2.

9. Brocklehurst P, Kujan O, O'Malley LA, Ogden G, Shepherd S, Glenny AM. Screening programmes for the early detection and prevention of oral cancer. Cochrane Database Syst Rev. 2013; 11: Art. No.: CD004150. DOI: 10.1002/14651858.CD004150.pub4.

10. NUK National Screening Committee- Oral Cancer in Screening in Adults http://www. screening.nhs.uk/oralcancer ( accessed June 2015)

Evidence-Based Dentistry (2015) 16, 46-47. doi:10.1038/sj.ebd.6401093 